\title{
Cantor Series Constructions Contrasting Two Notions of Normality
}

\author{
C. Altomare - B. Mance
}

Received: date / Accepted: date

\begin{abstract}
A. Rényi [1] made a definition that gives a generalization of simple normality in the context of $Q$-Cantor series. In [8], a definition of $Q$-normality was given that generalizes the notion of normality in the context of $Q$-Cantor series. In this work, we examine both $Q$-normality and $Q$-distribution normality, treated in [7] and [12. Specifically, while the non-equivalence of these two notions is implicit in [7], in this paper, we give an explicit construction witnessing the nontrivial direction. That is, we construct a base $Q$ as well as a real $x$ that is $Q$-normal yet not $Q$-distribution normal. We next approach the topic of simultaneous normality by constructing an explicit example of a base $Q$ as well as a real $x$ that is both $Q$-normal and $Q$-distribution normal.
\end{abstract}

Keywords Cantor series · Normal numbers · Uniform distribution

Mathematics Subject Classification (2000) 11K16 and 11A63

\section{Introduction}

Definition 1 Let $b$ and $k$ be positive integers. A block of length $k$ in base $b$ is an ordered $k$-tuple of integers in $\{0,1, \ldots, b-1\}$. A block of length $k$ is a

\author{
C. Altomare \\ Department of Mathematics \\ The Ohio State University \\ 231 West 18th Avenue \\ Columbus, OH 43210-1174 Tel.: (614) 292-1923 \\ Fax: (614) 292-1479 \\ E-mail: altomare@math.ohio-state.edu \\ B. Mance \\ Department of Mathematics \\ The Ohio State University \\ 231 West 18th Avenue \\ Columbus, OH 43210-1174 Tel.: (614) 292-6063 \\ Fax: (614) 292-1479 \\ E-mail: mance@math.ohio-state.edu
}


block of length $k$ in some base $b$. A block is a block of length $k$ in base $b$ for some integers $k$ and $b$.

Given a block $B,|B|$ will represent the length of $B$. Given non-negative integers $l_{1}, l_{2}, \ldots, l_{n}$, at least one of which is positive, and blocks $B_{1}, B_{2}, \ldots, B_{n}$, the block

$$
B=l_{1} B_{1} l_{2} B_{2} \ldots l_{n} B_{n}
$$

will be the block of length $l_{1}\left|B_{1}\right|+\ldots+l_{n}\left|B_{n}\right|$ formed by concatenating $l_{1}$ copies of $B_{1}, l_{2}$ copies of $B_{2}$, through $l_{n}$ copies of $B_{n}$. For example, if $B_{1}=(2,3,5)$ and $B_{2}=(0,8)$, then $2 B_{1} 1 B_{2} 0 B_{2}=(2,3,5,2,3,5,0,8)$.

Definition 2 Given an integer $b \geq 2$, the $b$-ary expansion of a real $x$ in $[0,1)$ is the (unique) expansion of the form

$$
x=\sum_{n=1}^{\infty} \frac{E_{n}}{b^{n}}=0 . E_{1} E_{2} E_{3} \ldots
$$

such that $E_{n}$ is in $\{0,1, \ldots, b-1\}$ for all $n$ with $E_{n} \neq b-1$ infinitely often.

Denote by $N_{n}^{b}(B, x)$ the number of times a block $B$ occurs with its starting position no greater than $n$ in the $b$-ary expansion of $x$.

Definition 3 A real number $x$ in $[0,1)$ is normal in base $b$ if for all $k$ and blocks $B$ in base $b$ of length $k$, one has

$$
\lim _{n \rightarrow \infty} \frac{N_{n}^{b}(B, x)}{n}=b^{-k}
$$

A number $x$ is simply normal in base $b$ if (3) holds for $k=1$.

Borel introduced normal numbers in 1909 and proved that almost all (in the sense of Lebesgue measure) real numbers in $[0,1)$ are normal in all bases. The best known example of a number that is normal in base 10 is due to Champernowne [3]. The number

$$
H_{10}=0.123456789101112 \ldots,
$$

formed by concatenating the digits of every natural number written in increasing order in base 10 , is normal in base 10 . Any $H_{b}$, formed similarly to $H_{10}$ but in base $b$, is known to be normal in base $b$. Since then, many examples have been given of numbers that are normal in at least one base. One can find a more thorough literature review in 4 and 6 .

The $Q$-Cantor series expansion, first studied by Georg Cantor, is a natural generalization of the $b$-ary expansion.

Definition $4 Q=\left\{q_{n}\right\}_{n=1}^{\infty}$ is a basic sequence if each $q_{n}$ is an integer greater than or equal to 2 . 
Definition 5 Given a basic sequence $Q$, the $Q$-Cantor series expansion of a real $x$ in $[0,1)$ is the (unique) expansion of the form

$$
x=\sum_{n=1}^{\infty} \frac{E_{n}}{q_{1} q_{2} \ldots q_{n}}
$$

such that $E_{n}$ is in $\left\{0,1, \ldots, q_{n}-1\right\}$ for all $n$ with $E_{n} \neq q_{n}-1$ infinitely often.

Clearly, the $b$-ary expansion is a special case of (4) where $q_{n}=b$ for all $n$. If one thinks of a $b$-ary expansion as representing an outcome of repeatedly rolling a fair $b$-sided die, then a $Q$-Cantor series expansion may be thought of as representing an outcome of rolling a fair $q_{1}$ sided die, followed by a fair $q_{2}$ sided die and so on. For example, if $q_{n}=n+1$ for all $n$, then the $Q$-Cantor series expansion of $e-2$ is

$$
e-2=\frac{1}{2}+\frac{1}{2 \cdot 3}+\frac{1}{2 \cdot 3 \cdot 4}+\ldots
$$

If $q_{n}=10$ for all $n$, then the $Q$-Cantor series expansion for $1 / 4$ is

$$
\frac{1}{4}=\frac{2}{10}+\frac{5}{10^{2}}+\frac{0}{10^{3}}+\frac{0}{10^{4}}+\ldots
$$

For a given basic sequence $Q$, let $N_{n}^{Q}(B, x)$ denote the number of times a block $B$ occurs starting at a position no greater than $n$ in the $Q$-Cantor series expansion of $x$. Additionally, define

$$
Q_{n}^{(k)}=\sum_{j=1}^{n} \frac{1}{q_{j} q_{j+1} \ldots q_{j+k-1}} .
$$

A. Rényi [11] defined a real number $x$ to be normal with respect to $Q$ if for all blocks $B$ of length 1 ,

$$
\lim _{n \rightarrow \infty} \frac{N_{n}^{Q}(B, x)}{Q_{n}^{(1)}}=1 .
$$

If $q_{n}=b$ for all $n$, then (6) is equivalent to simple normality in base $b$, but not equivalent to normality in base $b$. Thus, we want to generalize normality in a way that is equivalent to normality in base $b$ when all $q_{n}=b$.

Definition 6 A real number $x$ is $Q$-normal of order $k$ if for all blocks $B$ of length $k$,

$$
\lim _{n \rightarrow \infty} \frac{N_{n}^{Q}(B, x)}{Q_{n}^{(k)}}=1 .
$$

We say that $x$ is $Q$-normal if it is $Q$-normal of order $k$ for all $k$.

We make the following definitions: 
Definition 7 A basic sequence $Q$ is $k$-divergent if

$$
\lim _{n \rightarrow \infty} Q_{n}^{(k)}=\infty .
$$

$Q$ is fully divergent if $Q$ is $k$-divergent for all $k$.

Definition 8 A basic sequence $Q$ is infinite in limit if $q_{n} \rightarrow \infty$.

For $Q$ that are infinite in limit, it has been shown that the set of all $x$ in $[0,1)$ that are $Q$-normal of order $k$ has full Lebesgue measure if and only if $Q$ is $k$-divergent [11. Therefore if $Q$ is infinite in limit, then the set of all $x$ in $[0,1)$ that are $Q$-normal has full Lebesgue measure if and only if $Q$ is fully divergent. Additionally, given an arbitrary non-negative integer $a, \mathrm{~F}$. Schweiger [13] proved that for almost every $x$ with $\epsilon>0$, one has

$$
N_{n}((a), x)=Q_{n}^{(1)}+O\left(\sqrt{Q_{n}^{(1)}} \cdot \log ^{3 / 2+\epsilon} Q_{n}^{(1)}\right) .
$$

It is more difficult to construct specific examples of $Q$-normal numbers than it is to show that the typical real number is $Q$-normal. This is similar to the case of the $b$-ary expansion. The situation is more complicated when $Q$ is infinite in limit as we need to consider blocks whose digits come from an infinite set.

For example, normality can be defined for the continued fraction expansion, which involves an infinite digit set. While it is known that almost every real number is normal with respect to the continued fraction expansion, there are not many known examples (see [1] and [10]).

Generally speaking, it is more difficult to give explicit constructions of normal numbers (for various notions of normality) than it is to give typicality results. In [8], the second author gave an explicit construction of a basic sequence $Q$ and a real number $x$ such that $x$ is $Q$-normal. For the same $Q$ and $x$, we show that $x$ is $Q$-distribution normal, a term we now define. First, we must define $T_{Q, n}(x)$.

Definition 9 Let $x$ be a number in $[0,1)$ and let $Q$ be a basic sequence, then $T_{Q, n}(x)$ is defined as

$$
q_{1} \cdots q_{n} x \quad(\bmod 1) .
$$

Definition 10 A number $x$ in $[0,1)$ is $Q$-distribution normal if the sequence $\left\{T_{Q, n}(x)\right\}_{n=0}^{\infty}$ is uniformly distributed in $[0,1)$.

Note that in base $b$, where $q_{n}=b$ for all $n$, the notions of $Q$-normality and $Q$-distribution normality are equivalent. This equivalence is the most basic and fundamental fact in the study of normality in base $b$. It is surprising that this equivalence breaks down in the more general context of $Q$-Cantor series for general $Q$. 


\section{Block Friendly Families}

We will state a theorem that allows us to construct specific examples of $Q$ normal numbers for certain $Q$. We first need several definitions:

Definition 11 1 A weighting $\mu$ is a collection of functions $\mu^{(1)}, \mu^{(2)}, \mu^{(3)}, \ldots$ with $\sum_{j=0}^{\infty} \mu^{(1)}(j)=1$ such that for all $k, \mu^{(k)}:\{0,1,2, \ldots\}^{k} \rightarrow[0,1]$ and $\mu^{(k)}\left(b_{1}, b_{2}, \ldots, b_{k}\right)=\sum_{j=0}^{\infty} \mu^{(k+1)}\left(b_{1}, b_{2}, \ldots, b_{k}, j\right)$.

Definition 12 The uniform weighting in base $b$ is the collection $\lambda_{b}$ of functions $\lambda_{b}^{(1)}, \lambda_{b}^{(2)}, \lambda_{b}^{(3)}, \ldots$ such that for all $k$ and blocks $B$ of length $k$ in base $b$

$$
\lambda_{b}^{(k)}(B)=b^{-k}
$$

Definition 13 Let $p$ and $b$ be positive integers such that $1 \leq p \leq b$. A weighting $\mu$ is $(p, b)$-uniform if for all $k$ and blocks $B$ of length $k$ in base $p$, we have

$$
\mu^{(k)}(B)=\lambda_{b}^{(k)}(B)=b^{-k} .
$$

Given blocks $B$ and $y$, let $N(B, y)$ be the number of occurrences of the block $B$ in the block $y$.

Definition 14 Let $\epsilon$ be a real number such that $0<\epsilon<1$ and let $k$ be a positive integer. Assume that $\mu$ is a weighting. A block of digits $y$ is $(\epsilon, k, \mu)$ normal 2 if for all blocks $B$ of length $m \leq k$, we have

$$
\mu^{(m)}(B)|y|(1-\epsilon) \leq N(B, y) \leq \mu^{(m)}(B)|y|(1+\epsilon) .
$$

For the rest of the paper we use the following conventions freely and without comment. Given sequences of non-negative integers $\left\{l_{i}\right\}_{i=1}^{\infty}$ and $\left\{b_{i}\right\}_{i=1}^{\infty}$ with each $b_{i} \geq 2$ and a sequence of blocks $\left\{x_{i}\right\}_{i=1}^{\infty}$, we set

$$
\begin{gathered}
L_{i}=\left|l_{1} x_{1} \ldots l_{i} x_{i}\right|=\sum_{j=1}^{i} l_{j}\left|x_{j}\right| \\
q_{n}=b_{i} \text { for } L_{i-1}<n \leq L_{i},
\end{gathered}
$$

and

$$
Q=\left\{q_{n}\right\}_{n=1}^{\infty} .
$$

Moreover, if $\left(E_{1}, E_{2}, \ldots\right)=l_{1} x_{1} l_{2} x_{2} \ldots$, we set

$$
x=\sum_{n=1}^{\infty} \frac{E_{n}}{q_{1} q_{2} \ldots q_{n}} .
$$

Given $\left\{q_{n}\right\}_{n=1}^{\infty}$ and $\left\{l_{i}\right\}_{i=1}^{\infty}$, it is always assumed that $x$ and $Q$ are given by the formulas above. We make the following definition of a block friendly family $(\mathrm{BFF})$ :

\footnotetext{
1 [9] discusses normality in base 2 with respect to different weightings.

2 Definition 14 is a generalization of the concept of $(\epsilon, k)$-normality, originally due to Besicovitch [2].
} 
Definition $15 \mathrm{~A} B F F$ is a 6 -tuple $W=\left\{\left(l_{i}, b_{i}, p_{i}, \epsilon_{i}, k_{i}, \mu_{i}\right)\right\}_{i=1}^{\infty}$ with nondecreasing sequences of non-negative integers $\left\{l_{i}\right\}_{i=1}^{\infty},\left\{b_{i}\right\}_{i=1}^{\infty},\left\{p_{i}\right\}_{i=1}^{\infty}$ and $\left\{k_{i}\right\}_{i=1}^{\infty}$, for which $b_{i} \geq 2, b_{i} \rightarrow \infty$ and $p_{i} \rightarrow \infty$, such that $\left\{\mu_{i}\right\}_{i=1}^{\infty}$ is a sequence of $\left(p_{i}, b_{i}\right)$-uniform weightings and $\left\{\epsilon_{i}\right\}_{i=1}^{\infty}$ strictly decreases to 0 .

Definition 16 Let $W=\left\{\left(l_{i}, b_{i}, p_{i}, \epsilon_{i}, k_{i}, \mu_{i}\right)\right\}_{i=1}^{\infty}$ be a BFF. If $\lim k_{i}=K<$ $\infty$, then let $R(W)=\{0,1,2, \ldots, K\}$. Otherwise, let $R(W)=\{0,1,2, \ldots\}$. A sequence $\left\{x_{i}\right\}_{i=1}^{\infty}$ of $\left(\epsilon_{i}, k_{i}, \mu_{i}\right)$-normal blocks of non-decreasing length is said to be $W$-good if for all $k$ in $R$, the following three conditions hold:

$$
\begin{gathered}
\frac{b_{i}^{k}}{\epsilon_{i-1}-\epsilon_{i}}=o\left(\left|x_{i}\right|\right) ; \\
\frac{l_{i-1}}{l_{i}} \cdot \frac{\left|x_{i-1}\right|}{\left|x_{i}\right|}=o\left(i^{-1} b_{i}^{-k}\right) ; \\
\frac{1}{l_{i}} \cdot \frac{\left|x_{i+1}\right|}{\left|x_{i}\right|}=o\left(b_{i}^{-k}\right) .
\end{gathered}
$$

We now state a key theorem of [8].

Theorem 1 Let $W$ be a BFF and $\left\{x_{i}\right\}_{i=1}^{\infty}$ a $W$-good sequence. If $k \in R(W)$, then $x$ is $Q$-normal of order $k$. If $k_{i} \rightarrow \infty$, then $x$ is $Q$-normal.

\section{$3 Q$-Normality Without $Q$-Distribution Normality: A Construction}

In this section, we construct a specific example of a basic sequence $Q$ and a real number $x$ such that $x$ is $Q$-normal yet not $Q$-distribution normal. Moreover, the $Q$-distribution normality of $x$ fails in a particularly strong fashion. Not only does $\left\{T_{Q, n}(x)\right\}_{n=1}^{\infty}$ fail to be u.d. $\bmod 1$, but $\lim _{n \rightarrow \infty} T_{Q, n}(x)=0$.

We will use the following theorem of 12$]$ :

Theorem 2 Given a basic sequence $Q$ and a real number $x$ with $Q$-Cantor series expansion $x=\sum_{n=1}^{\infty} \frac{E_{n}}{q_{1} \cdots q_{n}}$; if

$$
\lim _{N \rightarrow \infty} \frac{1}{N} \sum_{n=1}^{N} \frac{1}{q_{n}}=0,
$$

then $x$ is $Q$-distribution normal iff

$$
\left\{\frac{E_{n}}{q_{n}}\right\}_{n=1}^{\infty}
$$

is u.d. $\bmod 1$. 
It should first be noted that it is easier to construct a basic sequence $Q$ and a real number $x$ that is $Q$-distribution normal, but not $Q$-normal. To see this, we let

$$
\begin{gathered}
\left(E_{1}, E_{2}, \ldots\right)=(1,1,2,1,2,3,1,2,3,4, \ldots) \text { and } \\
\left(q_{1}, q_{2}, \ldots\right)=(2,3,3,4,4,4,5,5,5,5, \ldots) .
\end{gathered}
$$

Thus, the number $x=\sum_{n=1}^{\infty} \frac{E_{n}}{q_{1} \ldots q_{n}}$ is not $Q$-normal since none of the digits $\left\{E_{n}\right\}$ are equal to 0 . However, $x$ is $Q$-distribution normal by Theorem 2 since the sequence $1 / 2,1 / 3,2 / 3,1 / 4,2 / 4,3 / 4,1 / 5, \ldots$ is $u . d$. $\bmod 1$.

The construction of a basic sequence $Q$ and a real number $x$ that is $Q$ normal but not $Q$-distribution normal is far more difficult. We will first need to define a sequence of weightings $\nu_{1}, \nu_{2}, \ldots$ and blocks $P_{b, w}$. After this, we will prove a number of technical lemmas from which the above stated facts follow.

If we let $b$ be a positive integer, then we define

$$
\nu_{b}^{(1)}(j)=\left\{\begin{array}{ll}
\frac{1}{2^{b}} & \text { if } 0 \leq j \leq b-1 \\
\frac{2^{b}-b}{2^{b}} & \text { if } j=b \\
0 & \text { if } j>b
\end{array} .\right.
$$

For a block $B=\left(b_{1}, \ldots, b_{k}\right)$, we define

$$
\nu_{b}^{(k)}(B)=\prod_{j=1}^{k} \nu_{b}^{(1)}\left(b_{j}\right) .
$$

Note that $\nu_{b}$ is a $\left(b, 2^{b}\right)$-uniform weighting. Since each $\nu_{b}^{(k)}$ is determined by $\nu_{b}^{(1)}$, we refer to $\nu_{b}^{(k)}$ as $\nu_{b}$ throughout.

Next, we define $P_{b, w}$. Let $b$ and $w$ be positive integers. Denote by $P_{1}, P_{2}, \ldots, P_{(b+1) w}$ the blocks in base $b+1$ of length $w$ written in lexicographic order. Let

$$
P_{b, w}=2^{b w} \nu_{b}\left(P_{1}\right) P_{1} 2^{b w} \nu_{b}\left(P_{2}\right) P_{2} \cdots 2^{b w} \nu_{b}\left(P_{(b+1) w}\right) P_{(b+1)} w .
$$

In order to get upper and lower bounds for $N\left(B, P_{b, w}\right)$ for a base $b+1$ block $B$, we need to calculate the length of $P_{b, w}$. We must first compute $\nu_{b}(B)$. This calculation is facilitated by the following definition:

Definition 17 Given a base $b+1$ block $B=\left(b_{1}, \ldots, b_{w}\right)$, set

$$
g_{b}(B)=\left|\left\{j ; b_{j}=b\right\}\right|
$$

Lemma 1 If $B$ is a base $b+1$ block of length $w$, then

$$
2^{b w} \nu_{b}(B)=\left(2^{b}-b\right)^{g_{b}(B)} .
$$


Proof

$$
2^{b w} \nu_{b}(B)=2^{b w} \cdot\left(\frac{2^{b}-b}{2^{b}}\right)^{g_{b}(B)} \cdot\left(\frac{1}{2^{b}}\right)^{w-g_{b}(B)}=\left(2^{b}-b\right)^{g_{b}(B)} .
$$

Lemma 2 If $b$ and $w$ are positive integers, then

$$
\left|P_{b, w}\right|=w \cdot 2^{b w} .
$$

Proof Fix $m$ such that $0 \leq m \leq w$. Clearly, the number of $i$ such that $g_{b}\left(P_{i}\right)=m$ is $\left(\begin{array}{c}w \\ m\end{array}\right) b^{w-m}$. By Lemma 1 and the definition of $P_{b, w}$, each block $P_{i}$ is concatenated $\left(2^{b}-b\right)^{m}$ times in forming $P_{b, w}$, with each one of these blocks having length $w$. It follows that the total number of digits contained in all copies of each block $P_{i}$ is

$$
w \cdot\left(\begin{array}{c}
w \\
m
\end{array}\right) \cdot\left(2^{b}-b\right)^{m} \cdot b^{w-m} .
$$

In order to obtain an expression for the length $\left|P_{b, w}\right|$ of $P_{b, w}$, we sum over all possible values of $m$. Therefore

$$
\left|P_{b, w}\right|=\sum_{m=0}^{w} w \cdot\left(\begin{array}{c}
w \\
m
\end{array}\right) \cdot\left(2^{b}-b\right)^{m} \cdot b^{w-m}=w \cdot\left(2^{b}-b+b\right)^{w}=w \cdot 2^{b w}
$$

by the binomial theorem.

Lemma 3 Let $w, k$, and $b$ be positive integers such that $k \leq w$. If $B$ is a block of length $k$ in base $b+1$, then

$$
N\left(B, P_{b, w}\right) \geq(w-k+1) \cdot\left(2^{b}-b\right)^{g_{b}(B)} \cdot 2^{b(w-k)} .
$$

Proof $P_{b, w}$ is defined as the concatenation of copies of the blocks $P_{i}=\left(p_{i, 1}, \ldots, p_{i, w}\right)$. In order to get this lower bound on $N\left(B, P_{b, w}\right)$ it is enough to show that the number of occurrences of $B$ inside some copy of some $P_{i}$ is exactly

$$
(w-k+1) \cdot\left(2^{b}-b\right)^{g_{b}(B)} \cdot 2^{b(w-k)} .
$$

Consider a block $P_{i}$ containing $B$. Since $B$ starts at position $s$ in $P_{i}$ for some $s$ such that $1 \leq s \leq w-k+1$, this leaves exactly $w-k$ digits of $P_{i}$ undetermined. Let

$$
M=\left\{j ; p_{i, j}=b \text { and } j \notin[s, s+k-1]\right\}
$$

and let $m=|M|$. Thus $m$ is the number of times that the block $P_{i}$ takes on the value $b$ outside of $B$. Clearly $0 \leq m \leq w-k$.

Note that since $m$ is the number of $b$ 's in $P_{i}$ outside $B$ and $g_{b}(B)$ is number of $b$ 's in $P_{i}$ inside $B$, we see that $g_{b}(B)+m$ is the total number of $b$ 's in $P_{i}$. By Lemma 1] exactly $\left(2^{b}-b\right)^{g_{b}(B)+m}$ copies of $P_{i}$ are concatenated in forming 
$P_{b, w}$. Let $S$ be the total number of occurrences of $B$ in blocks $P_{i}$ that have exactly $m$ occurrences of $b$ outside of $B$. Since there are $w-k+1$ choices for $s,\left(\begin{array}{c}w-k \\ m\end{array}\right)$ choices for $M, w-k-m$ undetermined positions after choosing $M$, and each undetermined position has $b$ possible values, we see that

$$
S=(w-k+1) \cdot\left(\begin{array}{c}
w-k \\
m
\end{array}\right) \cdot\left(2^{b}-b\right)^{g_{b}(B)+m} \cdot b^{w-k-m} .
$$

So, to count the number of times that $B$ occurs in $P_{b, w}$, we sum over $m$ from 0 to $w-k$ and use the binomial theorem to get

$$
\begin{gathered}
N\left(B, P_{b, w}\right) \geq \sum_{m=0}^{w-k}(w-k+1) \cdot\left(\begin{array}{c}
w-k \\
m
\end{array}\right) \cdot\left(2^{b}-b\right)^{g_{b}(B)+m} \cdot b^{w-k-m} \\
=(w-k+1) \cdot\left(2^{b}-b\right)^{g_{b}(B)} \sum_{m=0}^{w-k}\left(\begin{array}{c}
w-k \\
m
\end{array}\right) \cdot\left(2^{b}-b\right)^{m} \cdot b^{w-k-m} \\
=(w-k+1) \cdot\left(2^{b}-b\right)^{g_{b}(B)} \cdot\left(2^{b}\right)^{w-k} .
\end{gathered}
$$

We will need the following definition in the proof of Lemma 4 .

Definition 18 Let $B, C$, and $D$ be blocks with $|B| \geq 2$. Suppose that $B=$ $\left(b_{1}, \ldots, b_{k}\right), C=\left(c_{1}, \ldots, c_{m}\right)$, and $D=\left(d_{1}, \ldots, d_{t}\right)$. We say that $B$ straddles $C$ and $D$ if there is an integer $s$ in $[2, k]$, an integer $e$ in $[1, m]$, and an integer $f$ in $[1, \mathrm{t}]$ such that $\left(b_{1}, \ldots, b_{s-1}\right)=\left(c_{e}, \ldots, c_{m}\right)$ and $\left(b_{s}, \ldots, b_{k}\right)=\left(d_{1}, \ldots, d_{f}\right)$.

Intuitively, $B$ straddles $C$ and $D$ if $B$ starts in $C$ and ends in $D$. It is worth noting that with this definition, if $|B|=1$ then there are no choices of $C$ and $D$ for which $B$ straddles $C$ and $D$.

Lemma 4 Let $w, k$, and $b$ be positive integers such that $k \leq w$. If $B$ is a block of length $k$ in base $b+1$, then

$$
N\left(B, P_{b, w}\right) \leq w \cdot\left(2^{b}-b\right)^{g_{b}(B)} \cdot 2^{b(w-k)}+(k-1)(b+1)^{w} .
$$

Proof Note that $P_{b, w}$ has the form $1 C_{1} 1 C_{2} \cdots 1 C_{t}$ for some length $w$ blocks $C_{1}, \ldots, C_{t}$ and some $t$. In proving Lemma 3, we showed that the number of occurrences of $B$ in some $P_{i}$ is exactly

$$
(w-k+1) \cdot\left(2^{b}-b\right)^{g_{b}(B)} \cdot 2^{b(w-k)} .
$$

When (19) is added to an upper bound for the number of occurrences of $B$ in $P_{b, w}$ that straddle $C_{i}$ and $C_{i+1}$ for some $i$, we obtain an upper bound for $N\left(B, P_{b, w}\right)$.

Consider a block $B$ that straddles the block $C_{i}=\left(c_{i, 1}, \ldots, c_{i, w}\right)$ and $C_{i+1}=$ $\left(c_{i+1,1}, \ldots, c_{i+1, w}\right)$ for some $i$. In this case, $B$ starts at position $s$ in $C_{i}$ for some $s$ such that $w-k+2 \leq s \leq w$. Define $B_{1}=\left(c_{i, s}, \ldots, c_{i, w}\right), B_{2}=$ 
$\left(c_{i+1,1}, \ldots, c_{i+1, k-w+s-1}\right), B_{2}^{\prime}=\left(c_{i, 1}, \ldots, c_{i, k-w+s-1}\right)$, and $B_{1}^{\prime}=\left(c_{i+1, s}, \ldots, c_{i+1, w}\right)$.

Note that since $k \leq w$, these four sets are pairwise disjoint.

If $C_{i}=C_{i+1}$, then $B_{1}=B_{1}^{\prime}$ and $B_{2}=B_{2}^{\prime}$. Since the blocks $B_{1}$ and $B_{2}^{\prime}$ are both contained in $C_{i}$ and

$$
\left|B_{1}\right|+\left|B_{2}^{\prime}\right|=\left|B_{1}\right|+\left|B_{2}\right|=|B|=k,
$$

we see that $k$ positions of $C_{i}$ are determined. Thus there are $w-k$ undetermined positions in $C_{i}$.

Let

$$
M=\left\{j ; c_{i, j}=b \text { and } j \notin[k-w+s, s-1]\right\}
$$

and let $m=|M|$. Therefore $m$ is the number of times the block $C_{i}$ takes on the value $b$ outside $B_{2}^{\prime} \cup B_{1}$. We again note that $0 \leq m \leq w-k$.

Since $m$ is the number of $b$ 's in $C_{i}$ not determined by $B$, we know that $g_{b}\left(B_{1}\right)$ is number of $b$ 's in $C_{i}$ inside $B_{1}$ and $g_{b}\left(B_{2}^{\prime}\right)$ is number of $b$ 's in $C_{i}$ inside $B_{2}^{\prime}$. Thus

$$
g_{b}\left(C_{i}\right)=g_{b}\left(B_{1}\right)+g_{b}\left(B_{2}^{\prime}\right)+m=g_{b}\left(B_{1}\right)+g_{b}\left(B_{2}\right)+m=g_{b}(B)+m
$$

is the total of number of $b$ 's in $C_{i}$. By Lemma 1 it follows that exactly $\left(2^{b}-b\right)^{g_{b}(B)+m}$ copies of $C_{i}$ are concatenated in forming $P_{b, w}$. For a fixed $m$, define $S_{m}$ to be the total number of occurrences of $B$ straddling some $C_{i}$ and $C_{i+1}$ such that $C_{i}=C_{i+1}$ that have exactly $m$ occurrences of $b$ not determined by $B$. Since there are $k-1$ choices for $s,\left(\begin{array}{c}w-k \\ m\end{array}\right)$ choices for $M, w-k-m$ undetermined positions after choosing $M$ and each undetermined position has $b$ possible values, we see that for a fixed $m$

$$
S_{m} \leq(k-1) \cdot\left(\begin{array}{c}
w-k \\
m
\end{array}\right) \cdot\left(2^{b}-b\right)^{g_{b}(B)+m} \cdot b^{w-k-m} .
$$

To obtain an upper bound for the number of times $B$ occurs in $P_{b, w}$ straddling some $C_{i}$ and $C_{i+1}$ such that $C_{i}=C_{i+1}$, we need only sum over $m$ from 0 to $w-k$ and use the binomial theorem to get

$$
\begin{gathered}
S:=\sum_{m=0}^{w-k} S_{m} \leq(k-1) \sum_{m=0}^{w-k}\left(\begin{array}{c}
w-k \\
m
\end{array}\right) \cdot\left(2^{b}-b\right)^{g_{b}(B)+m} \cdot b^{w-k-m} \\
=\left(2^{b}-b\right)^{g_{b}(B)}(k-1) \sum_{m=0}^{w-k}\left(\begin{array}{c}
w-k \\
m
\end{array}\right)\left(2^{b}-b\right)^{m} b^{w-k-m}=(k-1)\left(2^{b}-b\right)^{g_{b}(B)}\left(2^{b}\right)^{w-k} .
\end{gathered}
$$

Next, we let $S^{\prime}$ be the number of occurrences of $B$ straddling the blocks $C_{i}$ and $C_{i+1}$ such that $C_{i}$ and $C_{i+1}$ are not equal. Let $Z$ denote the set of all $i$ such that $C_{i} \neq C_{i+1}$. Since the $C_{i}$ 's are written in lexicographic order, it follows that $Z$ has no more elements than the number of base $b+1$ blocks of length $w$. So $Z$ has at most $(b+1)^{w}$ elements. For each $i$ in $Z$, there are at most $k-1$ occurrences of $B$ straddling $C_{i}$ and $C_{i+1}$. Therefore

$$
S^{\prime} \leq(k-1) \cdot(b+1)^{w} .
$$


For each occurrence of $B$ in $P_{b, w}$, either $B$ occurs inside $C_{i}$ for some $i$, $B$ straddles some $C_{i}$ and $C_{i+1}$ for which $C_{i}=C_{i+1}$, or $B$ straddles some $C_{i}$ and $C_{i+1}$ for which $C_{i} \neq C_{i+1}$. We determined an upper bound for the number of occurrences of $B$ inside some $C_{i}$ in Lemma 3. In the proof of the current lemma, we showed that $S$ is an upper bound for the number of occurrences of $B$ straddling some $C_{i}$ and $C_{i+1}$ for which $C_{i}=C_{i+1}$. Also in the proof of the current lemma, we have seen that $S^{\prime}$ is an upper bound for the number of occurrences of $B$ straddling some $C_{i}$ and $C_{i+1}$ for which $C_{i} \neq C_{i+1}$. Putting these three facts together, we see that

$$
\begin{gathered}
N\left(B, P_{b, w}\right) \leq(w-k+1) \cdot\left(2^{b}-b\right)^{g_{b}(B)} \cdot 2^{b(w-k)}+S+S^{\prime} \\
\leq(w-k+1) \cdot\left(2^{b}-b\right)^{g_{b}(B)} \cdot 2^{b(w-k)}+(k-1) \cdot\left(2^{b}-b\right)^{g_{b}(B)} \cdot 2^{b(w-k)}+(k-1) \cdot(b+1)^{w} \\
=w \cdot\left(2^{b}-b\right)^{g_{b}(B)} \cdot 2^{b(w-k)}+(k-1) \cdot(b+1)^{w} .
\end{gathered}
$$

We now want to show that $P_{b, w}$ is $\left(\epsilon, k, \nu_{b}\right)$-normal. First we need a technical lemma:

Lemma 5 If $m, b, k$ and $w$ are positive integers such that $b \geq 6$ and $m \leq$ $k \leq w / 2$, then $(m-1)(b+1)^{w} \leq k \cdot 2^{b(w-m)}$.

Proof Since $m \leq k$ and $k \leq w / 2$, it follows that

$$
\begin{aligned}
& 1 \geq 2^{b(-w / 2+m)}=2^{-b w / 2} \cdot 2^{m b}=\left(2^{-b / 2}\right)^{w} \cdot 2^{m b} \\
& \geq\left((b+1) 2^{-b}\right)^{w} \cdot 2^{m b} \geq\left(\frac{m-1}{k}\right) \cdot \frac{(b+1)^{w} 2^{b m}}{2^{b w}},
\end{aligned}
$$

where (20) to (21) is due to $b+1 \leq 2^{b / 2}$ for $b \geq 6$. Therefore

$$
1 \geq\left(\frac{m-1}{k}\right) \cdot \frac{(b+1)^{w} 2^{b m}}{2^{b w}}
$$

Multiplying both sides of (22) by $k \cdot 2^{b(w-m)}$, the lemma follows.

Lemma 6 Let $b, k$ and $w$ be positive integers such that $b \geq 6$ and $k \leq w / 2$. If $\epsilon=\frac{k}{w}$, then $P_{b, w}$ is $\left(\epsilon, k, \nu_{b}\right)$-normal.

Proof By definition, $P_{b, w}$ is $\left(\epsilon, k, \nu_{b}\right)$-normal if for all blocks $B$ in base $b+1$ of length $m \leq k$

$$
\nu(B)\left|P_{b, w}\right|(1-\epsilon) \leq N\left(B, P_{b, w}\right) \leq \nu(B)\left|P_{b, w}\right|(1+\epsilon) .
$$

Therefore by Lemma 3 and Lemma 4, it is enough to show that

$$
\nu(B)\left|P_{b, w}\right|(1-\epsilon) \leq(w-m+1) \cdot\left(2^{b}-b\right)^{g_{b}(B)} \cdot 2^{b(w-m)}
$$


and

$$
w \cdot\left(2^{b}-b\right)^{g_{b}(B)} \cdot 2^{b(w-m)}+(m-1)(b+1)^{w} \leq \nu(B)\left|P_{b, w}\right|(1+\epsilon) .
$$

To show (24), we write

$$
\begin{gathered}
(1-\epsilon)\left|P_{b, w}\right| \nu_{b}(B)=\left(1-\frac{k}{w}\right) w \cdot 2^{b w}\left(2^{b}-b\right)^{g_{b}(B)} 2^{-b m} \\
=(w-k) \cdot\left(2^{b}-b\right)^{g_{b}(B)} \cdot 2^{b(w-m)}<(w-m+1) \cdot\left(2^{b}-b\right)^{g_{b}(B)} \cdot 2^{b(w-m)} .
\end{gathered}
$$

Next, to show (25), we write

$w \cdot\left(2^{b}-b\right)^{g_{b}(B)} \cdot 2^{b(w-m)}+(m-1)(b+1)^{w} \leq w \cdot\left(2^{b}-b\right)^{g_{b}(B)} \cdot 2^{b(w-m)}+k \cdot 2^{b(w-m)}$

$\leq w \cdot\left(2^{b}-b\right)^{g_{b}(B)} \cdot 2^{b(w-m)}+k \cdot\left(2^{b}-b\right)^{g_{b}(B)} \cdot 2^{b(w-m)}=(1+\epsilon) w \cdot\left(2^{b}-b\right)^{g_{b}(B)} \cdot 2^{b(w-m)}$,

where the first inequality follows from Lemma 5.

Theorem 3 For $i \leq 5$, let $x_{i}=(0,1), b_{i}=2$ and $l_{i}=0$. If for $i \geq 6$ we let $x_{i}=P_{i, i^{2}}, b_{i}=2^{i}$ and $l_{i}=2^{4 i^{2}}$, then $x$ is $Q$-normal.

Proof For each $i \geq 1$, we shall define numbers $p_{i}, k_{i}, \epsilon_{i}$, and weightings $\mu_{i}$ in order to define a $B F F W$ such that $\left\{x_{i}\right\}_{i=1}^{\infty}$ is $W$-good. Thus, we have only to verify (16), (17) and (18) of Theorem 1 to conclude that $x$ is $Q$-normal.

For $i \leq 5$, we define $p_{i}=2, k_{i}=1$ and $\mu_{i}=\lambda_{2}$. For $i \geq 6$, set $p_{i}=i$, $k_{i}=i$ and $\mu_{i}=\nu_{i}$. Define $\epsilon_{1}=.9, \epsilon_{1}=.8, \epsilon_{1}=.7, \epsilon_{1}=.6, \epsilon_{1}=.5$ and $\epsilon_{i}=1 / i$ for $i \geq 6$. Let $W=\left\{\left(l_{i}, b_{i}, p_{i}, \epsilon_{i}, k_{i}, \mu_{i}\right)\right\}_{i=1}^{\infty}$. We note that since $\mu_{i}$ is $\left(p_{i}, b_{i}\right)$-uniform, it follows by definition that $W$ is a $B F F$.

Since $\lim _{i \rightarrow \infty} k_{i}=\lim _{i \rightarrow \infty} i=\infty$, we see that $R(W)$ is the set of all nonnegative integers. So, it is enough to show that conditions (16), (17) and (18) hold for all non-negative integers $k$. First note that $\left|x_{i}\right|=i^{2} \cdot 2^{i^{3}}$ for $i \geq 6$.

To show (16), note that

$$
\lim _{i \rightarrow \infty}\left|x_{i}\right| /\left(\frac{2^{i k}}{\frac{1}{i-1}-\frac{1}{i}}\right)=\lim _{i \rightarrow \infty} \frac{i^{2} \cdot 2^{i^{3}}}{2^{i k} \cdot i(i-1)}=\infty .
$$

To show (17), notice that

$\lim _{i \rightarrow \infty} \frac{l_{i-1}}{l_{i}} \cdot \frac{\left|x_{i-1}\right|}{\left|x_{i}\right|} \cdot i \cdot 2^{i k} \leq \lim _{i \rightarrow \infty} 1 \cdot\left(\frac{i-1}{i}\right)^{2} \cdot \frac{2^{(i-1)^{3}+k i}}{2^{i^{3}}} \cdot i \leq \lim _{i \rightarrow \infty} 1 \cdot 2^{-3 i^{2}+(3+k) i-1} \cdot i=0$.

And finally, to show (18), we write

$$
\lim _{i \rightarrow \infty} \frac{1}{l_{i}} \cdot \frac{\left|x_{i+1}\right|}{\left|x_{i}\right|} \cdot 2^{i k}=\lim _{i \rightarrow \infty}\left(\frac{i+1}{i}\right)^{2} \cdot \frac{2^{(i+1)^{3}+k i}}{2^{4 i^{2}} \cdot 2^{i^{3}}} \leq \lim _{i \rightarrow \infty} 2 \cdot 2^{-i^{2}+(3+k) i+1}=0 .
$$

This shows that $\left\{x_{i}\right\}_{i=1}^{\infty}$ is $W$-good. Therefore $x$ is $Q$-normal by Theorem 1 . 
Theorem 4 If $\left\{x_{i}\right\},\left\{b_{i}\right\}$, and $\left\{l_{i}\right\}$ are defined as in Theorem 3 , then $\lim _{n \rightarrow \infty} T_{Q, n}(x)=$ 0 .

Proof To prove Theorem 4 we use the trick which is usually used to prove the irrationality of $x$. For more information see e.g. [5]. Note that

$$
T_{Q, n}(x)=q_{1} \cdots q_{n} x \quad(\bmod 1)=\frac{E_{n+1}}{q_{n+1}}+\frac{E_{n+2}}{q_{n+1} q_{n+2}}+\cdots
$$

Given $n$, define $j=j(n)$ as the unique integer satisfying

$$
L_{j-1}<n+1 \leq L_{j}
$$

Note that $q_{n+1}=b_{j}=2^{j}$ and $E_{n+1} \leq j$ by construction. Additionally, note that

$\frac{E_{n+2}}{q_{n+1} q_{n+2}}+\frac{E_{n+3}}{q_{n+1} q_{n+2} q_{n+3}}+\cdots \leq \frac{1}{q_{n+1}}\left[\frac{E_{n+2}}{q_{n+2}}+\frac{E_{n+3}}{q_{n+2} q_{n+3}}+\cdots\right] \leq \frac{1}{q_{n+1}} \cdot 1=\frac{1}{q_{n+1}}$.

Therefore since $0 \leq E_{n+1} \leq j$, we see that

$$
T_{Q, n}(x)=\frac{E_{n+1}}{q_{n+1}}+\left[\frac{E_{n+2}}{q_{n+1} q_{n+2}}+\frac{E_{n+3}}{q_{n+1} q_{n+2} q_{n+3}}+\cdots\right] \leq \frac{j}{2^{j}}+\frac{1}{2^{j}} \rightarrow 0 .
$$

Corollary 1 If $\left\{x_{i}\right\},\left\{b_{i}\right\}$, and $\left\{l_{i}\right\}$ are defined as in Theorem 3 , then $x$ is not $Q$-distribution normal.

\section{A Construction Giving Simultaneous $Q$-Normality and $Q$-Distribution Normality}

Definition 19 We say that $V=\left\{\left(l_{i}, b_{i}, \epsilon_{i}\right)\right\}_{i=1}^{\infty}$ is a modular friendly family $(M F F)$ if $\left\{l_{i}\right\}_{i=1}^{\infty}$ and $\left\{b_{i}\right\}_{i=1}^{\infty}$ are non-decreasing sequences of non-negative integers with $b_{i} \geq 2$ such that $\left\{\epsilon_{i}\right\}_{i=1}^{\infty}$ is a decreasing sequence of real numbers in $(0,1)$ with $\lim _{i \rightarrow \infty} \epsilon_{i}=0$.

Definition 20 Let $V=\left\{\left(l_{i}, b_{i}, \epsilon_{i}\right)\right\}_{i=1}^{\infty}$ be an $M F F$. A sequence $\left\{x_{i}\right\}_{i=1}^{\infty}$ of $\left(\epsilon_{i}, 1, \lambda_{b_{i}}\right)$-normal blocks of non-decreasing length with $\lim _{i \rightarrow \infty}\left|x_{i}\right|=\infty$ is said to be $V$-nice if the following two conditions hold:

$$
\begin{gathered}
\frac{l_{i-1}}{l_{i}} \cdot \frac{\left|x_{i-1}\right|}{\left|x_{i}\right|}=o(1 / i) ; \\
\frac{1}{l_{i}} \cdot \frac{\left|x_{i+1}\right|}{\left|x_{i}\right|}=o(1) .
\end{gathered}
$$


Throughout this section, we fix an $M F F V=\left\{\left(l_{i}, b_{i}, \epsilon_{i}\right)\right\}$ and a $V$-nice sequence of blocks $\left\{x_{i}\right\}$. Moreover, if $x_{i}=\left(x_{i, 1}, x_{i, 2}, \ldots, x_{i,\left|x_{i}\right|}\right)$, then $y_{i}$ will be understood to stand for the sequence

$$
\left\{\frac{x_{i, j}}{b_{i}}\right\}_{j=1}^{\left|x_{i}\right|} .
$$

Given finite sequences $y_{1}, \ldots, y_{t}$ and non-negative integers $l_{1}, \ldots, l_{t}$, the notation $l_{i} y_{i}$ denotes the concatenation of $l_{i}$ copies of $y_{i}$ and the notation $l_{1} y_{1} \ldots l_{t} y_{t}$ denotes the concatenation of the sequences $l_{1} y_{1}, \ldots, l_{t} y_{t}$.

Throughout the rest of the paper, for a given $n$, the letter $i=i(n)$ is the unique integer satisfying

$$
L_{i}<n \leq L_{i+1}
$$

Given a sequence $z=\left(z_{1}, \ldots, z_{n}\right)$ in $[0,1)$ and $0<\gamma \leq 1$, we define $A([0, \gamma), z)$ as

$$
\mid\left\{i ; 1 \leq i \leq n \text { and } z_{i} \in[0, \gamma)\right\} \mid .
$$

We recall the following standard definition:

Definition 21 For a finite sequence $z=\left(z_{1}, \ldots, z_{n}\right)$, we define the star discrepancy $D_{n}^{*}=D_{n}^{*}\left(z_{1}, \ldots, z_{n}\right)$ as

$$
\sup _{0<\gamma \leq 1}\left|\frac{A([0, \gamma), z)}{n}-\gamma\right| \text {. }
$$

Given an infinite sequence $w=\left(w_{1}, w_{2}, \ldots\right)$, we define

$$
D_{n}^{*}(w)=D_{n}^{*}\left(w_{1}, w_{2}, \ldots, w_{n}\right) .
$$

For convenience, set $D^{*}\left(z_{1}, \ldots, z_{n}\right)=D_{n}^{*}\left(z_{1}, \ldots, z_{n}\right)$. Obviously, this definition does not depend on the order that the $z_{i}$ 's are chosen in forming $z$. We will use this fact to reorder a sequence into an increasing sequence so that we may compute its star discrepancy with the following lemma from [6]:

Lemma 7 If $0 \leq z_{1} \leq \cdots \leq z_{n}<1$, then an upper bound for the star discrepancy $D_{n}^{*}\left(z_{1}, \ldots, z_{n}\right)$ is given by

$$
\frac{1}{2 n}+\max _{1 \leq i \leq n}\left|z_{i}-\frac{2 i-1}{2 n}\right|
$$

We note that by Lemma $7, \frac{1}{2 n} \leq D_{n}^{*}(z) \leq 1$ for all sequences $z=$ $\left(z_{1}, z_{2}, \ldots, z_{n}\right)$ with $z_{j}$ in $[0,1)$ for all $j$. It is well known that an infinite sequence $z=\left(z_{1}, \ldots, z_{n}, \ldots\right)$ is u.d. $\bmod 1$ iff $\lim _{n \rightarrow \infty} D_{n}^{*}\left(z_{1}, \ldots, z_{n}\right)=0$. This fact and Lemma 7 will allow us to prove $Q$-distribution normality of a well chosen $Q$ and $x$ by computing upper bounds on star discrepancies.

We recall the following lemma from [6]: 
Lemma 8 If $t$ is a positive integer and for $1 \leq j \leq t, z_{j}$ is a finite sequence in $[0,1)$ with star discrepancy at most $\epsilon_{j}$, then

$$
D^{*}\left(z_{1} z_{2} \cdots z_{t}\right) \leq \frac{\sum_{j=1}^{t}\left|z_{j}\right| \epsilon_{j}}{\sum_{j=1}^{t}\left|z_{j}\right|} .
$$

Corollary 2 If $t$ is a positive integer and for $1 \leq j \leq t, z_{j}$ is a finite sequence in $[0,1)$ with star discrepancy at most $\epsilon_{j}$, then

$$
D^{*}\left(l_{1} z_{1} \cdots l_{t} z_{t}\right) \leq \frac{\sum_{j=1}^{t} l_{j}\left|z_{j}\right| \epsilon_{j}}{\sum_{j=1}^{t} l_{j}\left|z_{j}\right|} .
$$

We note the following simple lemma:

Lemma 9 Let $U$ and $U^{\prime}$ be subsets of $\mathbb{R}$ such that $U$ has a maximum $M$ and a minimum $m$. If $f: U \rightarrow U^{\prime}$ is a monotone function, then $|f|$ has a maximum on $U$, which is either $f(m)$ or $f(M)$.

Proof Without loss of generality we may assume that $f$ is increasing. Therefore $f$ has a minimum at $m$ and a maximum at $M$. If $f(m) \geq 0$, then $f(x) \geq 0$ for all $x$ in $U$. This means that $|f|=f$ is increasing on $U$. Therefore $|f|$ attains a maximum at $M$. Similarly, if $f(M) \leq 0$, then $f(x) \leq 0$ for all $x$ in $U$. This implies that $|f|=-f$ is decreasing on $U$. Therefore $|f|$ attains a maximum at $m$.

The remaining case is that $f(m)<0<f(M)$. Let $U_{A}$ be the set of all $x$ in $U$ such that $f(x) \leq 0$ and let $U_{B}$ be the set of all $x$ in $U$ such that $f(x) \geq 0$. Note that $|f|$ is decreasing on $U_{A}$ and therefore $f \mid U_{A}$ has a maximum at $m$. Similarly, $|f|$ is increasing on $U_{B}$ and therefore $f \mid U_{B}$ has a maximum at $M$. Since $U=U_{A} \cup U_{B}$, it follows that $|f|$ has a maximum at $m$ or $M$.

Lemma 10 Let $x=\left(E_{1}, \ldots, E_{n}\right)$ be an $\left(\epsilon, 1, \lambda_{b}\right)$-normal block in base $b$. If $y=\left(E_{1} / b, \ldots, E_{n} / b\right)$, then

$$
D^{*}(y) \leq \frac{1}{b}+\epsilon+\frac{1}{|x|}
$$

Proof We wish to apply Lemma 7 to bound $D^{*}(y)$. However, Lemma 7 only applies to increasing sequences in $[0,1)$, so we must first reorder the sequence $y$. Let $z=\left(z_{1}, \ldots, z_{n}\right)$ be the sequence of values $E_{1} / b, \ldots, E_{n} / b$ written in increasing order. We note that each $z_{t}$ has the form $j / b$ for some $j$ in the set $\{0,1, \ldots, b-1\}$. Since $z$ is an increasing sequence, we may partition the integers from 1 to $n$ into intervals $U_{0}, \ldots, U_{b-1}$ such that $z_{t}=j / b$ for $t$ in $U_{j}$. We let $m_{j}$ and $M_{j}$ be the least and greatest elements of $U_{j}$, respectively.

By Lemma 7, we know that $D^{*}(z)$ is bounded above by

$$
\frac{1}{2 n}+\max _{1 \leq t \leq n}\left|z_{t}-\frac{2 t-1}{2 n}\right|
$$


Fix $j$. Note that $\frac{2 t-1}{2 n}$ is an increasing function of $t$ on $U_{j}$ and $z_{t}$ is a constant function of $t$ on $U_{j}$. Therefore $z_{t}-\frac{2 t-1}{2 n}$ is a decreasing function of $t$ on $U_{j}$. So, for each $j$, Lemma 9 shows that the expression $\left|z_{t}-\frac{2 t-1}{2 n}\right|$ is maximized for $t=m_{j}$ or $t=M_{j}$.

By Definition 14, we know that $x$ is $\left(\epsilon, 1, \lambda_{b}\right)$-normal iff for all $j$ in $0,1, \ldots, b-1$, we have

$$
(1-\epsilon) \frac{1}{b} n \leq N((j), x) \leq(1+\epsilon) \frac{1}{b} n .
$$

Thus,

$$
\left.m_{j}=\left(\sum_{t=0}^{j-1} N((t), x)\right)+1 \geq\left(\sum_{t=0}^{j-1}(1-\epsilon) \frac{1}{b} n\right)\right)+1=j(1-\epsilon) \frac{1}{b} n+1:=\bar{m}_{j}
$$

and

$$
M_{j}=\sum_{t=0}^{j} N\left((t, x) \leq \sum_{t=0}^{j}(1+\epsilon) \frac{1}{b} n=(j+1)(1+\epsilon) \frac{1}{b} n:=\bar{M}_{j} .\right.
$$

Letting

$$
f_{j}(x)=\left(\frac{j}{b}-\frac{2 x-1}{2 n}\right)
$$

we see that

$$
D^{*}(y) \leq \frac{1}{2 n}+\max _{1 \leq t \leq n}\left|z_{t}-\frac{2 t-1}{2 n}\right|=\frac{1}{2 n}+\max _{0 \leq j \leq b-1} \max \left(\left|f_{j}\left(m_{j}\right)\right|,\left|f_{j}\left(M_{j}\right)\right|\right) .
$$

Obviously, $f$ is a monotone function. Note that $\bar{m}_{j} \leq m_{j} \leq M_{j} \leq \bar{M}_{j}$. By Lemma 9, the maximum of $\left|f_{j}(x)\right|$ on $\left[\bar{m}_{j}, \bar{M}_{j}\right]$ occurs at $\bar{m}_{j}$ or $\bar{M}_{j}$. Therefore

$$
\max \left\{\left|f_{j}\left(m_{j}\right)\right|,\left|f_{j}\left(M_{j}\right)\right|\right\} \leq \max \left\{\left|f_{j}\left(\bar{m}_{j}\right)\right|,\left|f_{j}\left(\bar{M}_{j}\right)\right|\right\} .
$$

Note that

$$
\begin{gathered}
\left|f_{j}\left(\bar{m}_{j}\right)\right|=\left|\frac{j}{b}-\frac{2\left(j(1-\epsilon) \frac{1}{b} n+1\right)-1}{2 n}\right| \\
=\left|\frac{2 n j-2 j(1-\epsilon) n+b}{2 n b}\right|=\left|\frac{2 n j \epsilon+b}{2 n b}\right|=\frac{j \epsilon}{b}+\frac{1}{2 n} .
\end{gathered}
$$

Similarly, note that

$$
\begin{gathered}
\left|f_{j}\left(\bar{M}_{j}\right)\right|=\left|\frac{j}{b}-\frac{2(j+1)(1+\epsilon) \frac{1}{b} n-1}{2 n}\right| \\
=\left|\frac{2 n j-2 n j-2 n j \epsilon-2 n-2 n \epsilon+b}{2 n b}\right| \\
\leq\left|\frac{-2 n j \epsilon-2 n-2 n \epsilon}{2 n b}\right|+\left|\frac{b}{2 n b}\right|=\frac{j+1}{b} \epsilon+\frac{1}{b}+\frac{1}{2 n} .
\end{gathered}
$$


Thus $\max \left(\left|f_{j}\left(\bar{m}_{j}\right)\right|,\left|f_{j}\left(\bar{M}_{j}\right)\right|\right) \leq \frac{j+1}{b} \epsilon+\frac{1}{b}+\frac{1}{2 n}$ and we see that $D^{*}(y) \leq \frac{1}{2 n}+\max _{0 \leq j \leq b-1}\left(\frac{j+1}{b} \epsilon+\frac{1}{b}+\frac{1}{2 n}\right)=\frac{1}{2 n}+\left(\frac{b}{b} \epsilon+\frac{1}{b}+\frac{1}{2 n}\right)=\epsilon+\frac{1}{b}+\frac{1}{|x|}$.

By Lemma 10, we know that $D^{*}\left(y_{i}\right)$ is bounded above by

$$
\epsilon_{i}^{\prime}:=\frac{1}{b_{i}}+\epsilon_{i}+\frac{1}{\left|x_{i}\right|} \text {. }
$$

Given a positive integer $n$, let $m=n-L_{i}$. Note that $m$ can be written uniquely as $\alpha\left|x_{i+1}\right|+\beta$ with $0 \leq \alpha \leq l_{i+1}$ and $0 \leq \beta<\left|x_{i+1}\right|$. We define $\alpha$ and $\beta$ as the unique integers satisfying these conditions.

Let $y=l_{1} y_{1} l_{2} y_{2} \ldots$ and recall that $D^{*}(z)$ is bounded above by 1 for all finite sequences $z$ of real numbers in $[0,1)$. By Corollary 2 ,

$$
D_{n}^{*}(y) \leq f_{i}(\alpha, \beta):=\frac{l_{1}\left|x_{1}\right| \epsilon_{1}^{\prime}+\ldots+l_{i}\left|x_{i}\right| \epsilon_{i}^{\prime}+\left(\left|x_{i+1}\right| \epsilon_{i+1}^{\prime}\right) \alpha+\beta}{l_{1}\left|x_{1}\right|+\ldots+l_{i}\left|x_{i}\right|+\left|x_{i+1}\right| \alpha+\beta} .
$$

Note that $f_{i}(\alpha, \beta)$ is a rational function in $\alpha$ and $\beta$. We consider the domain of $f_{i}$ to be $\mathbb{R}_{0}^{+} \times \mathbb{R}_{0}^{+}$where $\mathbb{R}_{0}^{+}$is the set of all non-negative real numbers. Now we give an upper bound for $D_{n}^{*}(y)$. Since $D_{n}^{*}(y)$ is at most $f_{i}(\alpha, \beta)$, it is enough to bound $f_{i}(\alpha, \beta)$ from above on $\left[0, l_{i+1}\right] \times\left[0,\left|x_{i+1}\right|\right]$.

Lemma 11 If $l_{i}>0,\left|x_{i}\right|>0, \epsilon_{i+1}^{\prime}<1$,

$$
\begin{gathered}
l_{1}\left|x_{1}\right|+\ldots+l_{i-1}\left|x_{i-1}\right|>l_{1}\left|x_{1}\right| \epsilon_{1}^{\prime}+\ldots+l_{i-1}\left|x_{i-1}\right| \epsilon_{i-1}^{\prime}, \\
\frac{\left|x_{i+1}\right|}{l_{i}\left|x_{i}\right|}<\frac{1-\epsilon_{i}^{\prime}}{\epsilon_{i+1}^{\prime}}
\end{gathered}
$$

and

$$
(w, z) \in\left\{0, \ldots, l_{i+1}\right\} \times\left\{0, \ldots,\left|x_{i+1}\right|-1\right\}
$$

then

$$
f_{i}(w, z)<f_{i}\left(0,\left|x_{i+1}\right|\right)=\frac{l_{1}\left|x_{1}\right| \epsilon_{1}^{\prime}+\ldots+l_{i}\left|x_{i}\right| \epsilon_{i}^{\prime}+\left|x_{i+1}\right|}{l_{1}\left|x_{1}\right|+\ldots+l_{i}\left|x_{i}\right|+\left|x_{i+1}\right|} .
$$

Proof To bound $f_{i}(w, z)$, we first compute its partial derivatives $\frac{\partial f_{i}}{\partial z}(w, z)$ and $\frac{\partial f_{i}}{\partial w}(w, z)$. We will show that $\frac{\partial f_{i}}{\partial w}(w, z)$ is always negative, while $\frac{\partial f_{i}}{\partial z}(w, z)$ is always positive. Note that this is enough to prove Lemma 11 since $0 \leq \alpha$ and $\beta<\left|x_{i+1}\right|$.

First, we note that $f_{i}(w, z)$ is a rational function of $w$ and $z$ of the form

$$
f_{i}(w, z)=\frac{C+D w+E z}{F+G w+H z}
$$

where

$$
C=l_{1}\left|x_{1}\right| \epsilon_{1}^{\prime}+\ldots+l_{i}\left|x_{i}\right| \epsilon_{i}^{\prime}, D=\left|x_{i+1}\right| \epsilon_{i+1}^{\prime}, E=1,
$$




$$
F=l_{1}\left|x_{1}\right|+\ldots+l_{i}\left|x_{i}\right|, G=\left|x_{i+1}\right| \text { and } H=1 .
$$

Therefore

$$
\begin{aligned}
& \frac{\partial f_{i}}{\partial w}(w, z)=\frac{D(F+G w+H z)-G(C+D w+E z)}{(F+G w+H z)^{2}}=\frac{D(F+H z)-G(C+E z)}{(F+G w+H z)^{2}} \\
& \frac{\partial f_{i}}{\partial z}(w, z)=\frac{E(F+G w+H z)-H(C+D w+E z)}{(F+G w+H z)^{2}}=\frac{E(F+G w)-H(C+D w)}{(F+G w+H z)^{2}} .
\end{aligned}
$$

Thus, the sign of $\frac{\partial f_{i}}{\partial w}(w, z)$ does not depend on $w$ and the sign of $\frac{\partial f_{i}}{\partial z}(w, z)$ does not depend on $z$. We will show that $f_{i}(w, z)$ is a decreasing function of $w$ by proving that

$$
D(F+H z)<G(C+E z) .
$$

Similarly, we show that $f_{i}(w, z)$ is an increasing function of $z$ by verifying that

$$
E(F+G w)>H(C+D w) .
$$

Substituting the values in (31) into (32), we see that

$$
\left|x_{i+1}\right| \epsilon_{i+1}^{\prime}\left(l_{1}\left|x_{1}\right|+\ldots+l_{i}\left|x_{i}\right|+z\right)<\left|x_{i+1}\right|\left(l_{1}\left|x_{1}\right| \epsilon_{1}^{\prime}+\ldots+l_{i}\left|x_{i}\right| \epsilon_{i}^{\prime}+z\right) .
$$

Since $\left|x_{i+1}\right| \geq\left|x_{i}\right|>0$, we may divide both sides by $\left|x_{i+1}\right|$ to obtain

$$
l_{1}\left|x_{1}\right| \epsilon_{i+1}^{\prime}+\ldots+l_{i}\left|x_{i}\right| \epsilon_{i+1}^{\prime}+z \epsilon_{i+1}^{\prime}<l_{1}\left|x_{1}\right| \epsilon_{1}^{\prime}+\ldots+l_{i}\left|x_{i}\right| \epsilon_{i}^{\prime}+z .
$$

So, we only have to show (34), which is true since

$$
\epsilon_{i}^{\prime}=\frac{1}{b_{i}}+\epsilon_{i}+\frac{1}{\left|x_{i}\right|}
$$

is decreasing and $\epsilon_{i+1}^{\prime}<1$.

Also, by substituting the values in (31) into (33), we see that

$\left(l_{1}\left|x_{1}\right|+\ldots+l_{i-1}\left|x_{i-1}\right|\right)+\left(l_{i}\left|x_{i}\right|+w\left|x_{i+1}\right|\right)>\left(l_{1}\left|x_{1}\right| \epsilon_{1}^{\prime}+\ldots+l_{i-1}\left|x_{i-1}\right| \epsilon_{i-1}^{\prime}\right)+\left(l_{i}\left|x_{i}\right| \epsilon_{i}^{\prime}+\left|x_{i+1}\right| \epsilon_{i+1}^{\prime}\right)$.

By condition (29) we know that

$$
l_{1}\left|x_{1}\right|+\ldots+l_{i-1}\left|x_{i-1}\right|>l_{1}\left|x_{1}\right| \epsilon_{1}^{\prime}+\ldots+l_{i-1}\left|x_{i-1}\right| \epsilon_{i-1}^{\prime} .
$$

Therefore it is enough to show

$$
l_{i}\left|x_{i}\right|+w\left|x_{i+1}\right|>l_{i}\left|x_{i}\right| \epsilon_{i}^{\prime}+\left|x_{i+1}\right| \epsilon_{i+1}^{\prime} .
$$

Since $l_{i}\left|x_{i}\right|$ is the smallest possible value of $l_{i}\left|x_{i}\right|+w\left|x_{i+1}\right|$ for non-negative $w$, we need only show that

$$
l_{i}\left|x_{i}\right|>l_{i}\left|x_{i}\right| \epsilon_{i}^{\prime}+\left|x_{i+1}\right| \epsilon_{i+1}^{\prime} .
$$

By routine algebra, this is equivalent to

$$
\frac{\left|x_{i+1}\right|}{l_{i}\left|x_{i}\right|}<\frac{1-\epsilon_{i}^{\prime}}{\epsilon_{i+1}^{\prime}}
$$

which is true by (30). 
Set

$$
\bar{\epsilon}_{i}=f_{i}\left(0,\left|x_{i+1}\right|\right)=\frac{l_{1}\left|x_{1}\right| \epsilon_{1}^{\prime}+\ldots+l_{i}\left|x_{i}\right| \epsilon_{i}^{\prime}+\left|x_{i+1}\right|}{l_{1}\left|x_{1}\right|+\ldots+l_{i}\left|x_{i}\right|+\left|x_{i+1}\right|} .
$$

Lemma $12 \lim _{n \rightarrow \infty} \bar{\epsilon}_{i(n)}=0$.

Proof We write $i$ for $i(n)$ throughout. For $i$ large enough, we have

$$
\begin{aligned}
& \frac{l_{1}\left|x_{1}\right| \epsilon_{1}^{\prime}+\ldots+l_{i}\left|x_{i}\right| \epsilon_{i}^{\prime}+\left|x_{i+1}\right|}{l_{1}\left|x_{1}\right|+\ldots+l_{i}\left|x_{i}\right|+\left|x_{i+1}\right|}<\frac{l_{1}\left|x_{1}\right| \epsilon_{1}^{\prime}+\ldots+l_{i}\left|x_{i}\right| \epsilon_{i}^{\prime}+\left|x_{i+1}\right|}{l_{i}\left|x_{i}\right|} \\
= & \frac{l_{1}\left|x_{1}\right| \epsilon_{1}^{\prime}+\ldots+l_{i-1}\left|x_{i-1}\right| \epsilon_{i-1}^{\prime}}{l_{i}\left|x_{i}\right|}+\epsilon_{i}^{\prime}+\frac{\left|x_{i+1}\right|}{l_{i}\left|x_{i}\right|}<\frac{l_{i-1}\left|x_{i-1}\right|}{l_{i}\left|x_{i}\right|} \cdot i \cdot \epsilon_{i-1}^{\prime}+\epsilon_{i}^{\prime}+\frac{\left|x_{i+1}\right|}{l_{i}\left|x_{i}\right|},
\end{aligned}
$$

where the last inequality uses the fact that $\epsilon_{i}^{\prime}$ is decreasing. Note that $\frac{l_{i-1}\left|x_{i-1}\right|}{l_{i}\left|x_{i}\right|}$. $i \cdot \epsilon_{i-1}^{\prime} \rightarrow 0$ by (26),$\epsilon_{i}^{\prime}=\frac{1}{b_{i}}+\epsilon_{i}+\frac{1}{\left|x_{i}\right|} \rightarrow 0$ and $\frac{\left|x_{i+1}\right|}{l_{i}\left|x_{i}\right|} \rightarrow 0$ by (27). Therefore $\lim _{i \rightarrow \infty} \bar{\epsilon}_{i}=0$. Since $i$ can be made arbitrarily large by choosing large enough $n$, the lemma follows.

Theorem 5 If $V$ is an MFF and $\left\{x_{i}\right\}_{i=1}^{\infty}$ is a $V$-nice sequence, then $x$ is Q-distribution normal.

Proof By Theorem 2, it is enough to show that $D_{n}^{*}(y) \rightarrow 0$. Since $x_{i}$ is $\left(\epsilon_{i}, 1, \lambda_{b_{i}}\right)$-normal, we see that $D^{*}\left(y_{i}\right) \leq \epsilon_{i}^{\prime}$ by Lemma 10. We wish to apply Corollary 2 and for large enough $i$ apply Lemma 11 as well. To apply Lemma 11 for large $i$, we need only prove several inequalities for large $i$. In applying these inequalities, we will have $i=i(n)$ as defined in (28), so it is worth noting that $i$ may be chosen as large as one likes by choosing a large enough $n$.

For the first inequality, note that $\lim _{i \rightarrow \infty} l_{i}\left|x_{i}\right|=\infty$. For large enough $i$, the product $l_{i}\left|x_{i}\right|$ is nonzero. For the second, we have $\left|x_{i}\right|>0$. For the third inequality, $\epsilon_{i+1}^{\prime}<1$ for large enough $i$ as $\epsilon_{i}^{\prime} \rightarrow 0$. Next, since $l_{i-1}\left|x_{i-1}\right|$ asymptotically dominates $l_{i-1}\left|x_{i-1}\right| \epsilon_{i-1}^{\prime}$, it follows that $l_{1}\left|x_{1}\right|+\ldots+l_{i-1}\left|x_{i-1}\right|$ asymptotically dominates $l_{1}\left|x_{1}\right| \epsilon_{1}^{\prime}+\ldots+l_{i-1}\left|x_{i-1}\right| \epsilon_{i-1}^{\prime}$ as well. In particular, for large enough $i$, we have

$$
l_{1}\left|x_{1}\right|+\ldots+l_{i-1}\left|x_{i-1}\right|>l_{1}\left|x_{1}\right| \epsilon_{1}^{\prime}+\ldots+l_{i-1}\left|x_{i-1}\right| \epsilon_{i-1}^{\prime} .
$$

Finally, for the fifth inequality, noting that

$$
\lim _{i \rightarrow \infty} \frac{\left|x_{i+1}\right|}{l_{i}\left|x_{i}\right|}=0
$$

and that

$$
\lim _{i \rightarrow \infty} \frac{1-\epsilon_{i}^{\prime}}{\epsilon_{i+1}^{\prime}}=\infty
$$

since $\lim _{i \rightarrow \infty} \epsilon_{i}^{\prime}=0$, we see that

$$
\frac{\left|x_{i+1}\right|}{l_{i}\left|x_{i}\right|}<\frac{1-\epsilon_{i}^{\prime}}{\epsilon_{i+1}^{\prime}}
$$


for large $i$. 0 .

So, for large enough $i, D_{n}^{*}(y) \leq \bar{\epsilon}_{i}$ and $\lim _{i \rightarrow \infty} \bar{\epsilon}_{i}=0$. Thus $\lim _{n \rightarrow \infty} D_{n}^{*}(y)=$

Let $C_{b, w}$ be the block formed by concatenating all the blocks of length $w$ in base $b$ in lexicographic order. For example,

$$
\begin{gathered}
C_{3,2}=1(0,0) 1(0,1) 1(0,2) 1(1,0) 1(1,1) 1(1,2) 1(2,0) 1(2,1) 1(2,2) \\
=(0,0,0,1,0,2,1,0,1,1,1,2,2,0,2,1,2,2) .
\end{gathered}
$$

Let $x_{1}=(0,1), b_{1}=2$ and $l_{1}=0$. For $i \geq 2$, let $x_{i}=C_{i, i^{2}}, b_{i}=i$ and $l_{i}=i^{3 i}$. It was shown in 8 , that $x$ is $Q$-normal. We now show that $x$ is $Q$-distribution normal.

Theorem 6 Let $x_{1}=(0,1), b_{1}=2$ and $l_{1}=0$. If for $i \geq 2$, we let $x_{i}=C_{i, i^{2}}$, $b_{i}=i$, and $l_{i}=i^{3 i}$, then $x$ is $Q$-distribution normal.

Proof We let $\epsilon_{1}=3 / 5$. For $i \geq 2$, we let $\epsilon_{i}=1 / i$. By [8], we know that $x_{i}$ is $\left(\epsilon_{i}, 1, \lambda_{b_{i}}\right)$-normal. It is enough to show (26) and (27). Note that trivially, (17) implies (26) and (18) implies (27). Moreover, it was proven in 8] that (17) and (18) hold. Therefore $x_{i}$ is $V$-nice. So, by Theorem 5] we see that $x$ is $Q$-distribution normal as claimed.

Acknowledgements We thank the referees for their many valuable suggestions. We would also like to thank Vitaly Bergelson for pointing us in the direction of this problem and Marc Carnovale and Laura Harvey for their help in editing this paper.

\section{References}

1. Adler, R., Keane, M., Smorodinsky, M. : A construction of a normal number for the continued fraction transformation. J. Number Theory 13, 95-105 (1981)

2. Besicovitch, A. S. : The asymptotic distribution of the numerals in the decimal representation of the squares of the natural numbers. Math. Zeit. 39, 146-156 (1934)

3. Champernowne, D. G. : The construction of decimals normal in the scale of ten. Journal of the London Mathematical Society 8, 254-260 (1933)

4. Drmota, M., Tichy, R. F. : Sequences, Discrepancies and Applications. Springer-Verlag, Berlin Heidelberg (1997)

5. Hančl J., Tijdeman R. : On the irrationality of Cantor series, J. reine angew Math. 571, 145-158 (2004) 
6. Kuipers, L., Niederreiter, H. : Uniform Distribution of Sequences. Dover, Mineola, NY (2006)

7. Lafer, P. : Normal numbers with respect to Cantor series representation. Washington State University, Pullman, Washington (1974)

8. Mance, B. : Construction of normal numbers with respect to the Q-Cantor series representation for certain $Q$. submitted, available at http://arxiv.org/abs/0911.1485

9. Postnikov, A. G. : Ergodic problems in the theory of congruences and of diophantine approximations. Trudy Mat. Inst. Steklov 82 (1966); English transl. Proc. Steklov Inst. Math. 82 (1967).

10. Postnikov,A. G., Pyatetskii,I. I. : A Markov-sequence of symbols and a normal continued fraction. Izv. Akad. Nauk SSSR Ser. Mat. 21:6, 729-746 (1957)

11. Rényi,A. : On the distribution of the digits in Cantor's series. Mat. Lapok 7, 77-100 (1956)

12. S̆alát,T. : Zu einigen Fragen der Gleichverteilung (Mod 1). Czechoslovak Math. J. 18 (93), 476-488 (1968)

13. Schweiger, F. : Über den Satz von Borel-Rényi in der Theorie der Cantorschen Reihen. Monats. Math. 74, 150-153 (1969) 